\title{
Retos de las competencias digitales y alfabetización mediática para una educación abierta y en red
}

Challenges of digital competence and media literacy for open and online education

Desafios das competências digitais e alfabetização midiática para uma educação aberta e em rede

EDITORIAL

\section{Cecilia Ugalde}

Universidad del Azuay (Ecuador)

cugalde@uazuay.edu.ec

orcid.org/0000-0001-7716-6023

\section{Resumen:}

Frente a la creciente importancia de las competencias digitales y la alfabetización mediática, Obra Digital en su número 21 pretende abordar el tema desde enfoques y realidades diferentes de tal forma que se en este número se presenta un aporte que permite profundizar algunas áreas de este fascinante y cambiante realidad, con énfasis especial en la educación.

\section{PALABRAS CLAVE}

Competencia digital, Alfabetización mediática, Educación en red, TIC.

\section{Abstract:}

Given the growing importance of digital skills and media literacy, Obra Digital in its number 21 aims to address this issue from different approaches and realities in such a way that it presents a contribution that allows us to deepen some areas of this fascinating and chan- ging reality, with special emphasis on education.

\section{KEYWORDS}

Digital competence, Media literacy, Online education, ICTS.

\section{Resumo}

Diante da crescente importância das competências digitais e da alfabetização midiática, a Obra Digital no seu número 21 pretende abordar o tema a partir de diferentes abordagens e realidades de forma que este número apresente contribuições que permitam aprofundar algumas áreas desta fascinante e mutante realidade, com ênfase especial na educação.

\section{PALAVRAS-CHAVE}

Competência digital, Alfabetização midiática, Educação em rede, TIC. 
Las competencias digitales y la alfabetización mediática han venido cobrando importancia desde algunos años, como se puede observar en numerosos estudios (Gallardo et al., 2015; Gutiérrez-Martín \& Tyner, 2012; Kamerer, 2013; Potter, 1998), muchos de los cuales se han realizado en Iberoamérica (Ferrés Prats et al., 2012; Garro-Rojas, 2020; Mateus et al., 2020; Rivera-Rogel et al., 2017, 2019), y nos permiten tener una idea de la situación existente en la región. Sin embargo, la misma tecnología y sus avances, así como los cambios ocasionados por la crisis sanitaria global han resaltado no solo la importancia que las competencias digitales y la alfabetización mediática tienen en distintos entornos, sobre todo el educacional (Pattier, 2021; Thomas \& Rogers, 2020; Yan, 2021), sino, que dejan en evidencia la necesidad de mejorar las mismas para contar con ciudadanos mejor preparados para enfrentar los retos que la actualidad tecnológica y mediática presentan.

Aprobación y/o implementación de políticas públicas claras, así como inversión en equipos y mejor conectividad son algunas de las necesidades identificadas en algunos de los estudios citados y muchos otros que se han realizado sobre el tema (Cannon et al., 2020), pero de la mano de estas medidas, la capacitación docente es fundamental para mejorar las competencias digitales y la alfabetización mediática en los sistemas educativos, así como para disminuir la brecha digital existente entre maestros y estudiantes, (Gozálvez Pérez et al., 2014; Prensky \& Perry, 2001; Rivera et al., 2016), además de que aún hace falta mucha investigación en el área (Manca et al., 2021), razón por la que hacemos un aporte que permita seguir profundizando el tema, es así que Obra Digital en su número 21 presenta varias aristas y perspectivas en torno a las competencias digitales y la alfabetización mediática para una educación abierta y en red, como se puede observar en un análisis sobre la interacción entre estudiantes universitarios brasileños de diversas áreas con Zotero, y cómo esta herramienta contribuye a su alfabetización mediática. También se concluye en otro artículo que la influencia que el perfil socio familiar y el uso que estudiantes de educación obligatoria dan a la tecnología influyen positivamente en el desarrollo de la competencia digital en el área de la comunicación.

Y las condiciones de acceso, recursos y capacitación se evidencian en la competencia digital de los docentes, como se analiza en la brecha digital existente en la didáctica de los docentes de educación básica en escuelas públicas de Chihuahua, México, donde se identifican rasgos como el uso de tecnologías convencionales, bajo acceso a tecnologías para usos académicos y altas necesidades de aprendizaje, factores críticos que afectan la competencia digital de los maestros. Mientras que otro estudio realizado en Portugal analiza la competencia digital de profesores universitarios y de institutos politécnicos, donde se observa que características como trabajo a nivel doctoral, enseñanza online y la asociación con institutos politécnicos, se reflejan en niveles más altos de competencia digital.

También presentamos un análisis sistemático de la literatura sobre la integración de la tecnología en la educación secundaria portuguesa desde los años 70, en un intento para comprender la introducción de la tecnología a las escuelas en los últimos 50 años. Por otro lado, también se analizan las competencias digitales que necesitan los profesores, la brecha existente entre sus competencias profesionales y 
pedagógicas dejan en evidencia la necesidad de formación continua en el área.

Finalmente, la sección de misceláneas presenta dos artículos, uno sobre la relación entre el activismo medioambiental, el estilo de vida y las narrativas personales en Instagram de los "eco-influencers", y cerramos este número con una revisión de artículos publicados en los últimos cinco años sobre el rol de la persuasión narrativa en el edu-entretenimiento para mejorar la salud en América Latina, un tema que, dada la crisis sanitaria global, cobra especial importancia.
Así el número 21 de Obra Digital, correspondiente a los meses de septiembre 2021 - enero 2022, presenta ocho artículos enviados desde seis países (Brasil, Colombia, Ecuador, España, México, Portugal) de dos continentes. Los artículos se publican en sus versiones originales en español, portugués e inglés, además de una versión completa en inglés. Les invitamos a disfrutar de este número.

\section{REFERENCIAS}

Cannon, M., Connolly, S., \& Parry, R. (2020). Media literacy, curriculum and the rights of the child. Discourse: Studies in the Cultural Politics of Education, 1-13. https://doi.org/10.1080/01596306.2020.1829551

Ferrés Prats, J., Aguaded-Gómez, I., \& García-Matilla, A. (2012). La competencia mediática de la ciudadanía española: dificultades y retos. Revista ICONO14. Revista Científica de Comunicación y Tecnologías Emergentes, 10(3), 23-42. https://doi.org/10.7195/ri14.v10i3.201

Gallardo, E., Marqués, L., Minelli, J., \& Esteve, F. (2015). Digital competence in the knowledge society [La competencia digital en la sociedad del conocimiento]. MERLOT. Journal of Online Learning and Teaching, 11(1), 1-16.

Garro-Rojas, L. (2020). Alfabetización mediática en América Latina. Revisión de literatura: temas y experiencias. Revista Educación, 44(1), 520-532. https://doi.org/10.15517/revedu.v44i1.37708

Gozálvez Pérez, V., González Fernández, N., \& Caldeiro Pedreira, M. C. (2014). Media Lyteracy of Teachers: An Instrument for Assessing their Competence. Revista Electrónica de Investigación Educativa, 16(3), 129-146. http://www.redahttp//redie.uabc.mx/vol16no3/contenido-gonzalvez-glez-caldeiro.html\%OARevistalyc.org/articulo.oa?id=15532554009 
Gutiérrez-Martín, A., \& Tyner, K. (2012). Media Education, Media Literacy and Digital Competence. Comunicar, 19(38), 31-39. https://doi.org/10.3916/ C38-2012-02-03

Kamerer, D. (2013). Media Literacy. Communication Research Trends, 32(1), 4-32.

Manca, S., Bocconi, S., \& Gleason, B. (2021). "Think globally, act locally": A glocal approach to the development of social media literacy. Computers \& Education, 160, 104025. https://doi.org/10.1016/j.compedu.2020.104025

Mateus, J., Andrada, P., \& Quiroz, M.-T. (2020). Media Education in Latin America (J. Mateus, P. Andrada, \& M.-T. Quiroz (eds.)). Routledge.

Pattier, D. (2021). Referentes educativos durante la pandemia de la COVID-19: El éxito de los edutubers. PUBLICACIONES, 51(3), 533-563. https://doi.org/10.30827/publicaciones.v51i3.18080

Potter, W. J. (1998). Theory of Media Literacy: A Cognitive Approach. SAGE Publications.

Prensky, M., \& Perry, B. D. (2001). Diferentes tipos de experiencias llevan a diferentes tipos de estructuras cerebrales. En On the Horizon, 9(6), 1-9.

Rivera-Rogel, D., Mier, C., Rodríguez-Hidalgo, C., Andrade, L., Iriarte, M., Marín-Gutiérrez, I., Beltrán, A. M., Velásquez, A., Mendoza-Zambrano, D., Ugalde, C., González-Cabrera, C., Carrión, G., Celly, S., Torres-Toukoumidis, Á., Ortiz, F., \& Freire, R. (2019). Libro blanco. Competencias mediátics en Ecuador. Pearson.

Rivera-Rogel, D., Zuluaga-Arias, L. I., Ramírez, N. M. M., Romero-Rodríguez, L. M., Aguaded, I., Rivera, D., Zuluaga, L. I., Ramírez, N. M. M., Romero-Rodríguez, L. M., \& Aguaded, I. (2017). Media Competencies for the Citizenship Training of Teachers from Andean America: Colombia and Ecuador. Paidéia (Ribeirão Preto), 27(66), 80-89. https://doi. org/10.1590/1982-43272766201710

Rivera, D., Ugalde, C. (Universidad del A., González-Cabrera, C. (Universidad del A., Carrión, G., González, C., Salinas, G. C., González-Cabrera, C. (Universidad del A., \& Carrión, G. (2016). Uso que profesores y estudiantes ecuatorianos dan a las Tecnologías de la Información y la Comunicación. Espacios, 37(№33), 2.

Thomas, M. S. C., \& Rogers, C. (2020). Education, the science of learning, and the COVID-19 crisis. Prospects, 49(1-2), 87-90. https://doi.org/10.1007/ s11125-020-09468-z

Yan, S. (2021). COVID-19 and technology use by teenagers: A case study. Human Behavior and Emerging Technologies, 3(1), 185-193. https://doi. org/10.1002/hbe2.236 